\title{
Linewidth reduction and frequency stabilization of a semiconductor laser with a combination of FM sideband locking and optical feedback
}

\author{
Y. Shevy, ${ }^{*}$ J. Kitching, and A. Yariv \\ Department of Applied Physics, California Institute of Technology, M/S 128-95, Pasadena, California 91125
}

Received February 3, 1993

\begin{abstract}
We describe a novel method for semiconductor laser noise reduction that uses a combination of optical and electronic feedback. A Doppler-free Faraday resonance in Cs vapor provided both optical feedback and discrimination for an electronic feedback scheme incorporating FM sideband spectroscopy. The introduction of electronic feedback further reduced the low-frequency fluctuation noise power by more than 2 orders of magnitude, resulting in a linewidth of $1.4 \mathrm{kHz}$.
\end{abstract}

In previous papers we reported the reduction of semiconductor laser FM noise through the use of dispersive loss. ${ }^{1-4}$ We demonstrated a $10-\mathrm{kHz}$ semiconductor laser linewidth by using optical feedback from a Doppler-free Faraday resonance in Cs vapor. It was also shown that the linewidth reduction was limited by the $1 / f$ component of the frequencyfluctuation noise spectrum $S_{\dot{\varphi}}(f)$. With our optical feedback scheme both the $1 / f$ and the white components of the frequency-fluctuation spectrum were reduced by more than 5 orders of magnitude. However, since the laser linewidth is linearly paratus (see Fig. 1) consists of a Cs vapor cell between crossed linear polarizers that is subjected to a weak axial magnetic field. This system is placed inside an external cavity formed by the semiconductor front facet and a reflector placed $\sim 40 \mathrm{~cm}$ away. This arrangement produces weak optical feedback into the semiconductor laser cavity through narrow, Doppler-free reflection at the Cs transition.

The FM noise reduction that is due to the optical feedback only, assuming that the laser frequency with optical feedback coincides with the free-running laser frequency (zero frequency pulling), is given by ${ }^{2}$

$$
S_{\dot{\varphi}}(f)=\frac{S_{\dot{\varphi}}{ }^{0}(f)}{Q^{2}} \equiv \frac{S_{\dot{\varphi}}{ }^{0}(f)}{\left\{1+\sqrt{1+\alpha^{2}}\left[\kappa(\omega)\left(\tau_{0}+\partial \phi^{\prime} / \partial \omega\right) \cos \left(\phi+\tan ^{-1} \alpha\right)\right]\right\}^{2}},
$$

proportional to the white-noise power while it scales as the square root of the $1 / f$ noise power, the laser's linewidth was reduced by a factor of only 2000 .

This result suggests that, if the $1 / f$ noise component were eliminated, the semiconductor laser linewidth would approach the already-low whitenoise line-width. This objective is tangible since the $1 / f$ noise in semiconductor lasers usually extends only to a few hundred kilohertz. In this Letter we describe the addition of electronic feedback to the optically locked semiconductor laser in order to reduce the $1 / f$ noise further. We have chosen the FM sideband scheme introduced by Drever et al. ${ }^{5}$ owing to its superior signal-to-noise ratio and large bandwidth. Our preliminary results indicate an additional reduction of the low-frequency $1 / f$ noise by more than 2 orders of magnitude. Our best result to date is a field spectrum with a FWHM of $1.4 \mathrm{kHz}$ and improvement of the laser's Allen variance, at integration times above $1 \mathrm{~ms}$, by an order of magnitude from the case of optical feedback alone. We achieved this result by utilizing the same resonance used for the optical feedback in a relatively compact apparatus.

The Doppler-free Faraday resonance scheme was described in detail in our previous Letter. ${ }^{2}$ The ap- where $S_{\dot{\varphi}}{ }^{0}(f)$ is the laser's frequency-fluctuation spectrum without feedback, $\alpha$ is the amplitude-to-phase

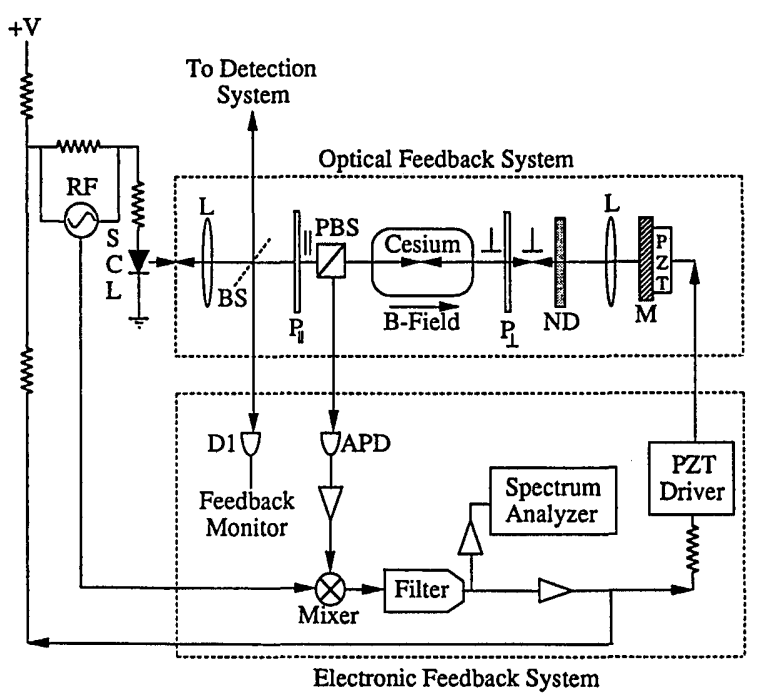

Fig. 1. Experimental setup: SCL, semiconductor laser cavity; L's, lenses; BS, beam splitter; PBS, polarizing beam splitter; ND, neutral-density filter; $M$, mirror; PZT's, piezoelectric transducers; P's, polarizers; D1, photodiode; APD, avalanche photodiode. 


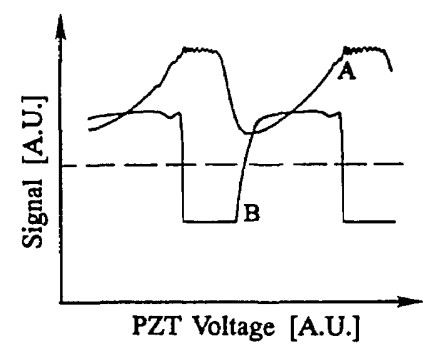

Fig. 2. Optical feedback intensity (curve A) and the electronic feedback error signal (curve B) versus the laser frequency. We obtained this trace by varying the PZT voltage (note that because of chirp reduction the horizontal scale is not linear with frequency ${ }^{2}$ ).

coupling factor, $\kappa(\omega)$ and $\phi^{\prime}(\omega)$ are, respectively, the frequency-dependent feedback coupling rate and the phase shift that are due to the Cs vapor, and $\tau_{0}$ is the external-cavity round-trip time without the Cs cell.

We showed previously that this system produces the maximum reduction in the laser linewidth at the top of the Cs resonance, where the slope of the dispersion, $\partial \phi^{\prime}(\omega) / \partial \omega$, that results from the Cs resonance is maximum. We have also found that because of the narrow spectral width of $\phi^{\prime}(\omega)$ one can obtain $Q$ 's of the order of $10^{3} .^{2,3}$ It was also confirmed that both the white-noise and the $1 / f$ noise power are reduced by a factor of $Q^{2}$, while the $1 / f$ contribution to the linewidth is reduced by only $Q{ }^{4}$ In the following we show experimentally that the inclusion of an electronic feedback mechanism to both the feedback phase and the injection current can reduce the $1 / f$ noise beyond that with optical feedback only. Thus the linewidth approaches its fundamental limit that is due to the white-noise only.

In the experiments described here, we used an STC Model LT50A-03U laser. This laser has a threshold current of $50 \mathrm{~mA}$, was operated single mode at $120 \mathrm{~mA}$ with a power of $85 \mathrm{~mW}$, and was collimated to a spot size of $0.5 \mathrm{~cm}^{2}$. The higher laser power resulted in a larger power broadening of the Cs resonance (compared with that in our previous experiments with a Hitachi HLP 1400 laser), which reduced $Q$ in the present conditions to a value of $\sim 100$.

The FM sideband locking technique is shown in Fig. 1. To generate the FM sidebands, we modulated the laser injection current. Since the laser was under conditions of optical feedback, it experienced chirp reduction, which reduced the amplitude of the FM sidebands by a factor of $Q$. The laser was therefore modulated at a frequency of $28.7 \mathrm{MHz}$, which corresponds to a multiple of the system response time. This resulted in an enhancement of the FM modulation, creating a first-order FM sideband containing $\sim 1 \%$ of the laser power. The reflected probe beam with the polarization orthogonal to the laser had an intensity of $1 \mathrm{~mW}$ and was collected with a polarizing beam splitter and focused into an avalanche photodiode. The amplified beat-note signal was fed into a doubly balanced mixer, with the 28.7-MHz modulating signal serving as the local oscillator. With the appropriate delay between the beatnote signal and the local oscillator, one can observe at the output of the mixer a frequency deviation error signal with a typical dispersive line shape. Figure 2 shows the line shape of the optical feedback and the FM sideband error signal, indicating that one can obtain an absolute electronic frequency discriminator with the zero-crossing locking point coinciding with the top of the optical feedback.

Figure 3(a), trace A, shows the spectrum of the FM error signal, measured with an electronic spectrum analyzer, as the laser was tuned to the top of the Faraday resonance. A $1 / f$ error signal with a signal-to-noise ratio of more than $20 \mathrm{~dB}$ was obtained at $\mathrm{dc}$, and a signal-to-noise ratio of $10 \mathrm{~dB}$ was obtained at higher frequencies (relative to the noise floor the discriminator). The error signal was fed to the external mirror piezoelectric transducer drive in order to stabilize the optical feedback phase. The mechanical resonances of this mirror limited the servo bandwidth to frequencies below a few kilohertz. In Fig. 3(a), trace B, we show a reduction of the low-frequency noise power by more than $20 \mathrm{~dB}$. Further improvement in the signal-to-noise ratio and the servo network would give a better result. To gain access to the higher-frequency noise, we applied the FM error signal to the laser's injection current. Figure 3(a), trace $B$, shows a reduction of the FM noise at higher frequencies by more than $10 \mathrm{~dB}$. This reduction was again limited by the noise floor of the discriminator.

As can be clearly seen, in trace B of Fig. 3(a) the $1 / f$ noise was drastically reduced and the noise power approached a white spectrum. The large peaks at low frequencies are $60-\mathrm{Hz}$-line frequency harmonics

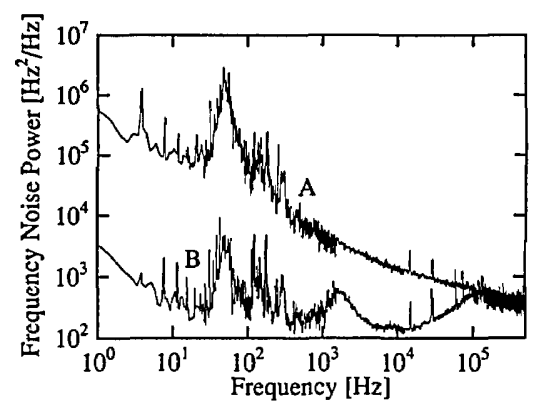

(a)

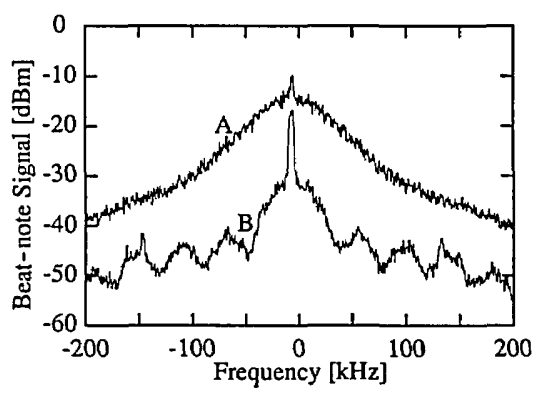

(b)

Fig. 3. (a) Frequency noise spectral density and (b) the self-heterodyne beat-note signal. Curves $A$ are obtained with optical feedback only, and curves B are with a combination of optical and electronic feedback. The ripples in Fig. 3(b), trace B, are an indication that the laser linewidth is below the resolution of our self-heterodyne apparatus $(6 \mathrm{kHz})$. 


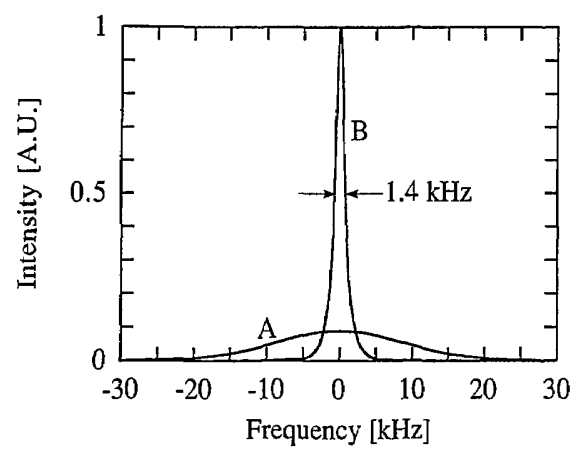

Fig. 4. Field spectrum calculated with the data from Fig. 3(a) with optical feedback only (trace A) and a combination of optical and electronic feedback (trace B).

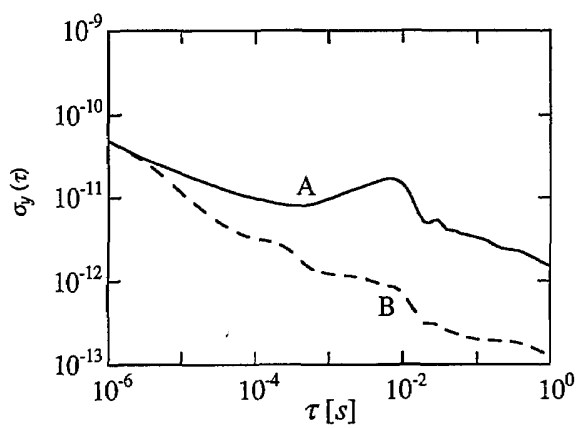

Fig. 5. Square root of the Allen variance calculated from Fig. 3(a) with optical feedback only (trace A) and a combination of optical and electronic feedback (trace B).

and mechanical resonances of the external cavity. We calibrated the noise power by comparing it with the frequency noise measured using a reflection mode of a Fabry-Perot cavity. The calibration was double checked with a self-heterodyne measurement. Since the linewidth of the laser with electronic feedback, as shown in trace B of Fig. 3(b), was below the resolution limit of the self-heterodyne system, we used the line shape of the laser with the optical feedback only [Fig. 3(b), trace A]. Owing to the large $1 / f$ noise contribution to the linewidth, we fitted this spectrum to a Lorentzian raised to the power $3 / 2{ }^{2,4}$

To find the laser linewidth with both electronic and optical feedback, we numerically calculated the field spectrum of the laser $I(\nu)$, using the following relations between this quantity and a single-sided frequency noise power spectrum $S_{\dot{\varphi}}(f)$ in units of $\mathrm{Hz}^{2} / \mathrm{Hz}$ obtained from Fig. 3(a):

$$
\begin{aligned}
I(\nu)= & 4 \operatorname{Re} \int_{-\infty}^{\infty} \exp \left[2 \pi i\left(\nu-\nu_{0}\right) \tau\right] \\
& \times \exp \left[-2(\pi \tau)^{2} \sigma^{2}(\tau)\right] \mathrm{d} \tau,
\end{aligned}
$$

where

$$
\sigma^{2}(\tau)=\int_{0}^{\infty} S_{f}(f) \frac{\sin ^{2}(\pi f \tau)}{(\pi f \tau)^{2}} \mathrm{~d} f
$$

The calculated field spectrum of the laser under the conditions of optical feedback and both optical and electronic feedback is shown in Fig. 4. The FWHM of the field spectrum with the addition of electronic feedback is $1.4 \mathrm{kHz}$. This is more than an order-ofmagnitude improvement over the result with optical feedback only $(20 \mathrm{kHz})$.
Using the noise power spectrum of Fig. 3(a), we also calculated the Allen variance of the laser $\sigma_{y}(\tau)$, given by

$$
\sigma_{y}^{2}(\tau)=\frac{2}{\nu_{0}^{2}} \int_{0}^{\infty} S_{f}(f) \frac{\sin ^{4}(\pi f \tau)}{(\pi f \tau)^{2}} \mathrm{~d} f .
$$

Figure 5 displays the outcome again with optical feedback and both electronic and optical feedback. A reduction of the Allen variance to a value of $\sim 10^{-13}$ at an integration time of $1 \mathrm{~Hz}$ is achieved. This is an order of magnitude better than with the optical feedback.

These preliminary results represent the best values, to our knowledge, for a semiconductor laser locked directly to an atomic resonance. Lee and Campbell ${ }^{6}$ also demonstrated a combination of optical and electronic feedback in $\mathrm{Rb}$ vapor and obtained a linewidth of $500 \mathrm{kHz}$. Recently Nakagawa et al. ${ }^{7}$ independently demonstrated the utility of the FM sideband technique for semiconductor laser linewidth reduction by using a reflection mode from a cavity. The frequency stability of this system, however, depends on the mechanical stability of the reference cavity.

In conclusion, we have demonstrated a novel technique for linewidth reduction and absolute frequency stabilization of a semiconductor laser locked to an atomic transition. Using a Doppler-free Faraday resonance to provide both optical feedback and FM sideband discrimination for electronic feedback, we were able to reduce the semiconductor laser linewidth from $10 \mathrm{MHz}$ down to $1.4 \mathrm{kHz}$. The electronic feedback also improved the laser's Allen variance by a factor of 10 over that with optical feedback only.

This research was supported by the Defense Advanced Research Project Agency, the U.S. Office of Naval Research, and the U.S. Air Force Office of Scientific Research. J. Kitching thanks the National Science and Engineering Research Council of Canada for financial support.

*Present address, Department of Physics, University of Miami, Box 248046, Coral Gables, Florida 33124 .

\section{References}

1. A. Yariv, R. Nabiev, and K. Vahala, Opt. Lett. 15, 1359 (1990).

2. Y. Shevy, J. Iannelli, J. Kitching, and A. Yariv, Opt. Lett. 17, 661 (1992).

3. J. Iannelli, Y. Shevy, J. Kitching, and A. Yariv, "Linewidth reduction and frequency stabilization of semiconductor lasers using dispersive losses in an atomic vapor," IEEE J. Quantum Electron. (to be published).

4. J. Kitching, Y. Shevy, J. Iannelli, and A. Yariv, "Measurements of $1 / f$ frequency noise reduction in semiconductor lasers using optical feedback with dispersive loss," J. Lightwave Technol. (to be published).

5. R. Drever, J. Hall, F. Kowalsky, J. Hough, G. Ford, M. Manley, and H. Ward, Appl. Phys. B 31, 97 (1983).

6. W. D. Lee and J. C. Campbell, Appl. Phys. Lett. 60, 1544 (1992).

7. N. Nakagawa, M. Kurogi, and M. Ohtsu, Opt. Lett. 17, 934 (1992). 